\title{
Radio-Frequency Tracking of Respiratory Equipment: Rationale and Early Experience at the Cleveland Clinic
}

\author{
James K Stoller MD MSc FAARC, Vincent Roberts RRT, David Matt, Leslie Chom, \\ Madhu Sasidhar MD, and Robert L Chatburn MHHS RRT-NPS FAARC
}

\begin{abstract}
BACKGROUND: When respiratory therapists (RTs) seek respiratory care equipment, finding it quickly is desirable, both to expedite patient care and to avert RTs wasting time. To optimize RTs' ability to quickly locate ventilators, we developed and implemented a radio-frequency identification (RFID) tagging system called eTrak. METHODS: The Clinical Engineering and Information Technology groups at Cleveland Clinic collaboratively developed a Wi-Fi-based RFID program that used active RFID tags. Altogether, 218 ventilators, 82 noninvasive ventilators, and various nonrespiratory equipment were tagged, beginning in March 2010. We calculated the difference in time required to locate equipment before versus after implementation. RESULTS: The eTrak system had a mean 145 log-ons per week over the first year of use, and was associated with a decreased time required for RTs to locate ventilators: median $18 \mathrm{~min}$ (range 1-45 min) versus $3 \mathrm{~min}$ (range 1-6 min) $(P<.001)$. Surveys of RTs regarding whether equipment was hard to find before versus after implementing eTrak showed a non-significant trend toward improvement. CONCLUSIONS: An RFID tracking system for respiratory equipment shortened the time to locate ventilators and non-significantly improved RT satisfaction with finding equipment. RFID tagging of equipment warrants further investigation. Key words: radio-frequency identification; RFID; real-time locating systems; indoor tracking; equipment tracking. [Respir Care 2013;58(12):2069-2075. () 2013 Daedalus Enterprises]
\end{abstract}

\section{Introduction}

Radio-frequency identification (RFID) is the use of a wireless non-contact system that uses radio-frequency electromagnetic fields to transfer data from a tag attached to an object for the purposes of automatic identification and tracking. A passive RFID tag requires no battery, and is powered and read at short range via a magnetic field (electromagnetic induction). An active RFID tag uses battery power to emit radio-frequency wave. The tag contains

The authors are affiliated with the Respiratory Institute, Cleveland Clinic Foundation, 9500 Euclid Avenue, M56, Cleveland OH 44195.

The authors have disclosed no conflicts of interest.

Correspondence: Robert L Chatburn MHHS RRT-NPS FAARC, Respiratory Institute, Cleveland Clinic Foundation, 9500 Euclid Avenue, M56, Cleveland OH 44195. E-mail: chatbur@ccf.org.

DOI: $10.4187 /$ respcare. 02545 electronically stored information that can be read from up to several yards away. ${ }^{1}$

RFID tracking of equipment and/or inventory is widely used in various business sectors and in the military to ensure product/equipment availability and delivery, minimize loss, and facilitate business processes (eg, supply chain management). ${ }^{1-4}$ For example, based on the military origins of RFID for distinguishing friendly from hostile aircraft, more recent uses have involved active tracking of radioactive materials during transport. ${ }^{2}$ Similarly, large companies such as Wal-Mart use RFID devices for inventory control.

Like all large hospital systems, Cleveland Clinic faces the challenge of locating respiratory care equipment (especially ventilators) and other commonly used devices (eg, intravenous pumps) over a large main hospital and a larger multi-hospital care system. To address this challenge, we recently designed and implemented a system (called eTrak) that tracks respiratory equipment with RFID tags. Issues that prompted us to develop an RFID system to track 
equipment throughout the Cleveland Clinic Health System included:

- Difficulty locating equipment delays patient care.

- When RTs spend undue time finding equipment, RT time is wasted, which harms RT morale and engagement.

- There can be an erroneous assumption that misplaced equipment is permanently lost, potentially causing unnecessary expenditure for replacement.

- Locating equipment easily and quickly is critically important in a mass-casualty scenario and aligns with the cost-containment imperative for greater operational efficiency in all healthcare institutions.

- RFID tagging facilitates preventive maintenance on equipment.

Here we describe the eTrak system and report our early metrics of its use and impact, and some lessons from that use.

\section{Methods}

\section{Design of the eTrak System}

The eTrak RFID tracking system was developed collaboratively by the Clinical Engineering and Information Technology groups at Cleveland Clinic. Guiding principles that drove the design and that led to in-house design were maintaining flexibility in developing the feature set, and controlling costs.

The initiative began by evaluating various RFID technologies: infrared, ultrasound, ZigBee, and wireless technologies based on the 802.11 standard (Wi-Fi). Based on an evaluation of the costs, capabilities, accuracy, and longterm supportability of the platform, we chose the existing wireless network infrastructure and decided to base future equipment location efforts on the Wi-Fi platform. We used the existing network infrastructure as our backbone, to supplement it as needed with compatible technologies to deliver room-level location, and to build and extend the user interface based on ongoing feedback from our clinical users. This approach allowed us to get a workable equipment-tracking system to learn from with a minimal investment up front. Because other institutional strategies (mobile workstations, voice-over-internet protocol) had paved the way for a wireless equipment-tracking system, our infrastructure costs were limited to the application server and database. To provide the resiliency and performance needed for this critical clinical application, we extended our existing hosting environment. Our investment was just over $\$ 100,000$, as we were able to take advantage of enterprise licensing and a virtual server infrastructure.

\section{QUICK LOOK}

\section{Current knowledge}

Radio-frequency identification (RFID) is the use of a wireless non-contact system to transfer data from a tag attached to an object for the purposes of automatic identification and tracking. RFID tracking of equipment and inventory is widely used in various sectors to ensure product/equipment availability and delivery, and to minimize loss.

\section{What this paper contributes to our knowledge}

Implementation of an RFID system for respiratory equipment was associated with shortened time for respiratory therapists to locate ventilators, and with a trend toward enhanced therapist satisfaction with finding equipment.

Internal resources were redeployed to focus on the initial build-out of the eTrak system; it took a cross-functional team of Web developers, business analysts, network engineers, and project managers 9 months to iterate through what was desirable and match that with what was possible. Through negotiation, an inventory of RFID tags with various capabilities was acquired, for an average cost of $\$ 60$ per tag. Given the experimental nature of our program, these costs were not charged back to the departments that used the tags.

The eTrak system allows RTs to log onto a Web site that shows a map of the current location of all the tracked equipment (Fig. 1). Because equipment is characterized in eTrak by its type, model, and features, the system allows users to locate specific types of equipment (eg, a specific ventilator model with specific features).

Core functional components of the eTrak system include:

- User log-on and user management

- Equipment location

- Device administration

- Floor view of facilities

- Ability to query for particular types of devices

- Reporting

- Online help system and documentation

Noteworthy features of eTrak include:

- The ability to remember the device and location that the user last accessed. For clinicians who routinely access only items on their floor or a particular type of equipment, this greatly simplifies the interface. The user sim- 


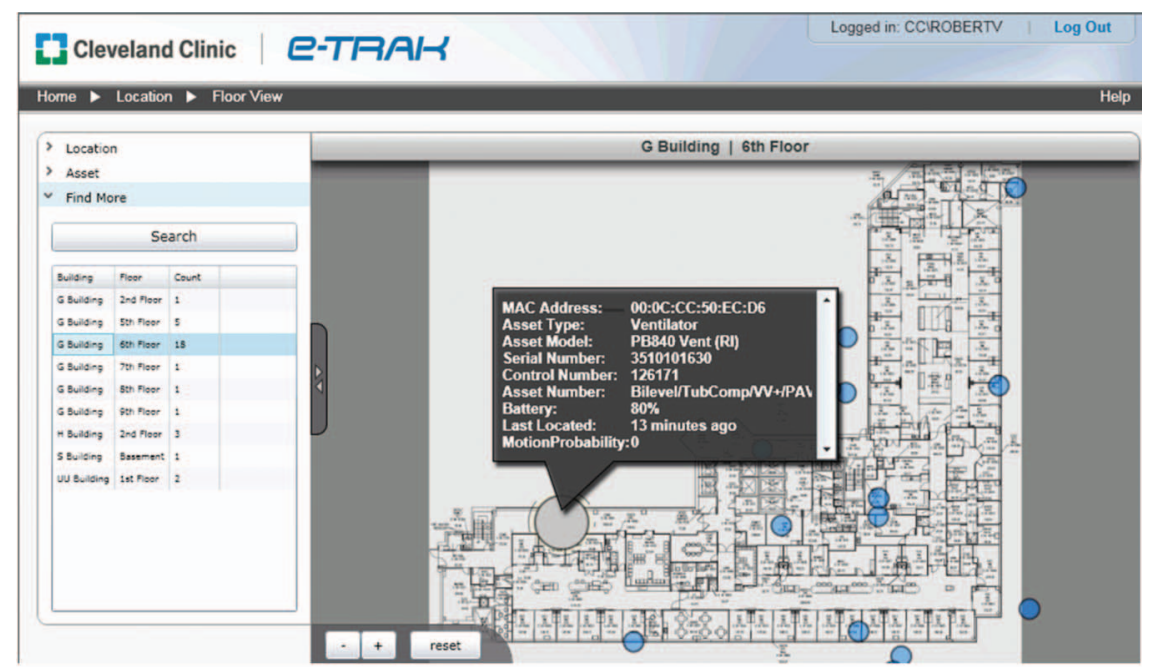

Fig. 1. Screenshot of the eTrak system display as it appears to a respiratory therapist seeking a piece of equipment. The screen identifies the type of equipment, identifying information (eg, serial number), and information about the battery duration of the radio-frequency identification tag, allowing preventive maintenance to avoid battery failure that would cause the equipment to disappear from view.

ply logs on and sees the floor they routinely access. The system also permits a search of the entire campus through a "Search All" feature.

- The ability to control who can see a particular category of assets. For instance, all healthcare providers who are designated as "respiratory therapists" (RTs) can access all equipment in the ventilator/CPAP category. There are currently 300 pieces of RT equipment, in 43 equipment categories.

- Real-time calculation of the RFID position on the map, scaled and plotted to the display screen. The screen can be panned and zoomed without flicker or other graphical detractors.

- The ability to "find more" of a particular asset. For instance, if a patient requires the particular capabilities of a particular ventilator type, the RT can locate other similar ventilators in the hospital.

The RFID tags are strategically placed on each piece of equipment to prevent loss or damage to the tags. The preferred method of securing the tags was with a heavyduty zip tie in a discreet but easily accessible location (Fig. 2A). If no such location existed (ie, small CPAP unit), a heavy-duty Velcro-like product (Dual Lock, 3M, St Paul, Minnesota) was used (see Fig. 2B). This has proved to be the strongest method for attaching the tag if a zip tie could not be used. Both methods allow easy tag removal and battery replacement. Care is taken to affix the tag to the machine itself, and not to a stand or cart. This prevents equipment from being placed on a cart with an incorrect RFID tag.

\section{Results}

\section{Early Experience Using the eTrak System}

One impetus for implementing an RFID system was widespread RT dissatisfaction about the time spent searching for equipment, as demonstrated by low employee engagement scores (using the Gallup employee engagement tool, Gallup, Washington DC) regarding the question: "I have the materials and equipment I need to do my work." Fifty percent of respondents responded "no." Further inquiry by a focused survey in RT departments indicated that inability to locate ventilators was responsible for most of the expressed dissatisfaction, with up to $20 \%$ of respondents indicating that equipment was too hard to find.

\section{Design and Roll-Out of the eTrak System}

The Cleveland Clinic Health System consists of a main campus hospital and 8 regional hospitals in northeast Ohio. In implementing eTrak at all the Cleveland Clinic Health System hospitals, we undertook to tag 218 ventilators and 82 noninvasive ventilators with active RFID tags.

Based on the institutional need to implement a system, our requirements for the eTrak system were refined over the course of 2010, and the first release went live in March 2010. We used RFID tags from AeroScout (Redwood City, California, see Fig. 2). Tagging the respiratory devices was undertaken by a team of RTs and was completed over 8 weeks. Non-respiratory devices (eg, deaf talk machines, computers on wheels, intravenous pumps, wound vacuum units, rental beds and equipment, and various patient mon- 

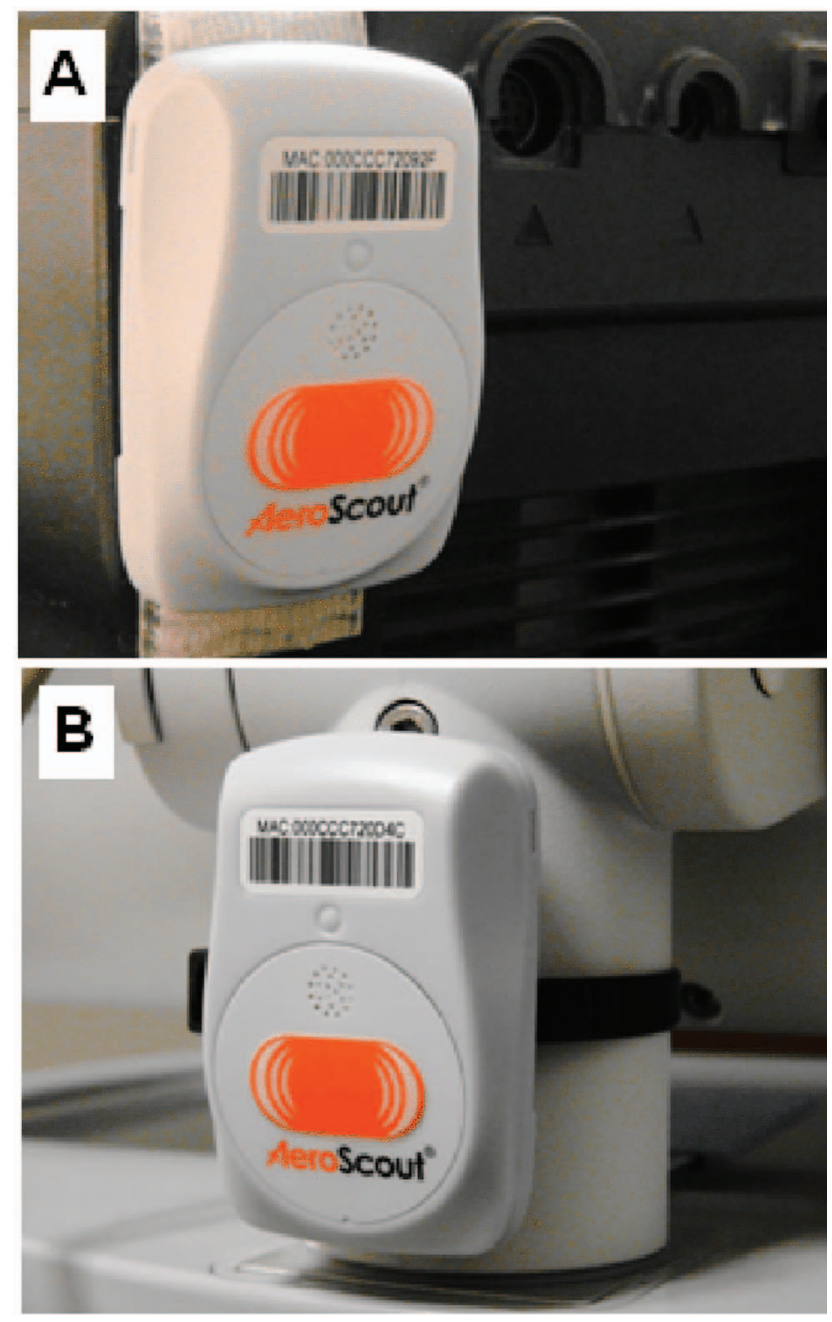

Fig. 2. Radio-frequency identification tag. A: Attachment with Dual Lock. B: Attachment with zip tie.

itors) were also tagged. Altogether, more than 8,000 items were tagged for tracking 135 types of equipment.

The implementation of the eTrak system was announced with a series of in-service talks to potential users, and a one-page eTrak training document and training video were prepared and made available.

\section{Impact of the eTrak System}

Over the period from June 2012 to February 2013 we recorded a mean $\pm \mathrm{SD} 145 \pm 2$ weekly log-ons to the eTrak system (Fig. 3). The number peaked at $202 \log$-ons/ week during the influenza season, and was lowest (64 log-ons/week) in December 2012. Individual log-ons may have involved finding multiple devices, and individuals may have made multiple log-ons.

Baseline (pre-implementation) measurement included the time required to find a ventilator (ie, the time from the

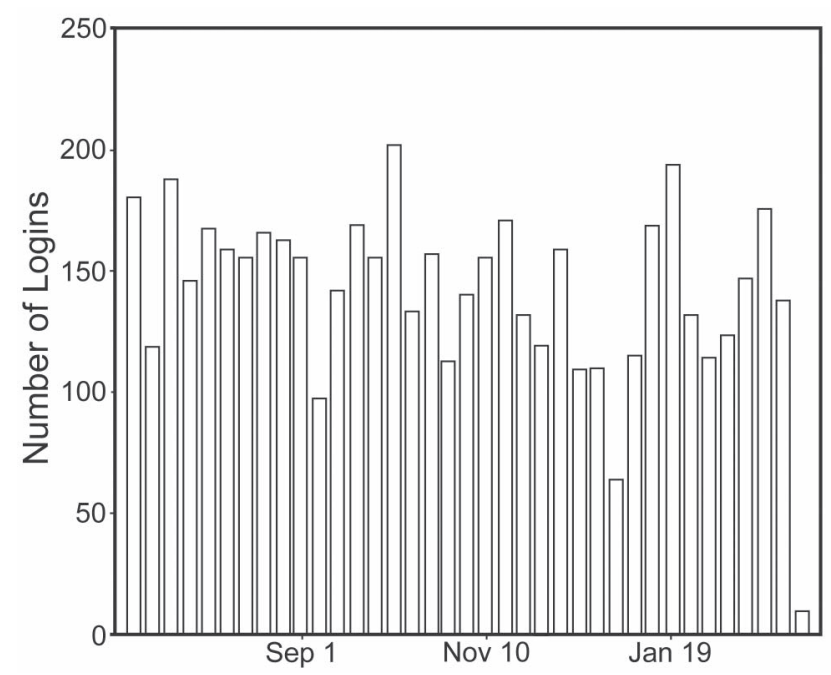

Fig. 3. Number of weekly log-ons to eTrak, June 30, 2012, to February 28, 2013.

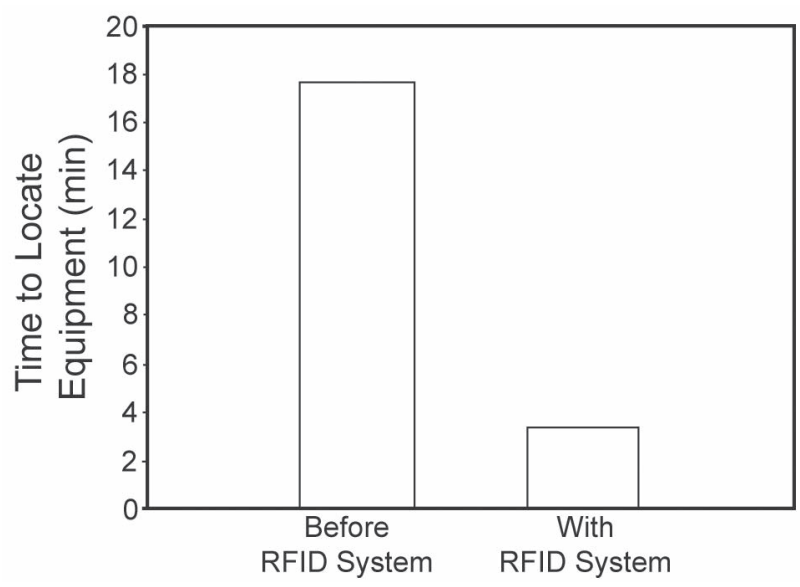

Fig. 4. Median time between when the respiratory therapist began to look for a ventilator and found it, before versus after implementing the eTrak radio-frequency identification (RFID) equipment tracking system.

RT's being notified of the need for the equipment and actually physically procuring it), which was undertaken by a survey of 25 staff RTs (including supervisors) at the Cleveland Clinic main hospital (of 300 total main campus RTs, $8 \%$ ). These RTs agreed to prospectively log the aforementioned times, which were collected and analyzed before and after eTrak installation. The eTrak system was associated with a significant shortening of the RT time needed to locate ventilators (Fig. 4): median 18 min (range $1-45 \mathrm{~min})$ versus $3 \mathrm{~min}$ (range $1-6 \mathrm{~min})(P<.001 \mathrm{using}$ the Mann Whitney rank sum test).

To assess the impact of eTrak on RTs' perceptions regarding equipment availability, 2 respiratory care departments at the Cleveland Clinic main campus (in the Anesthesia and Respiratory Institutes, respectively) were 


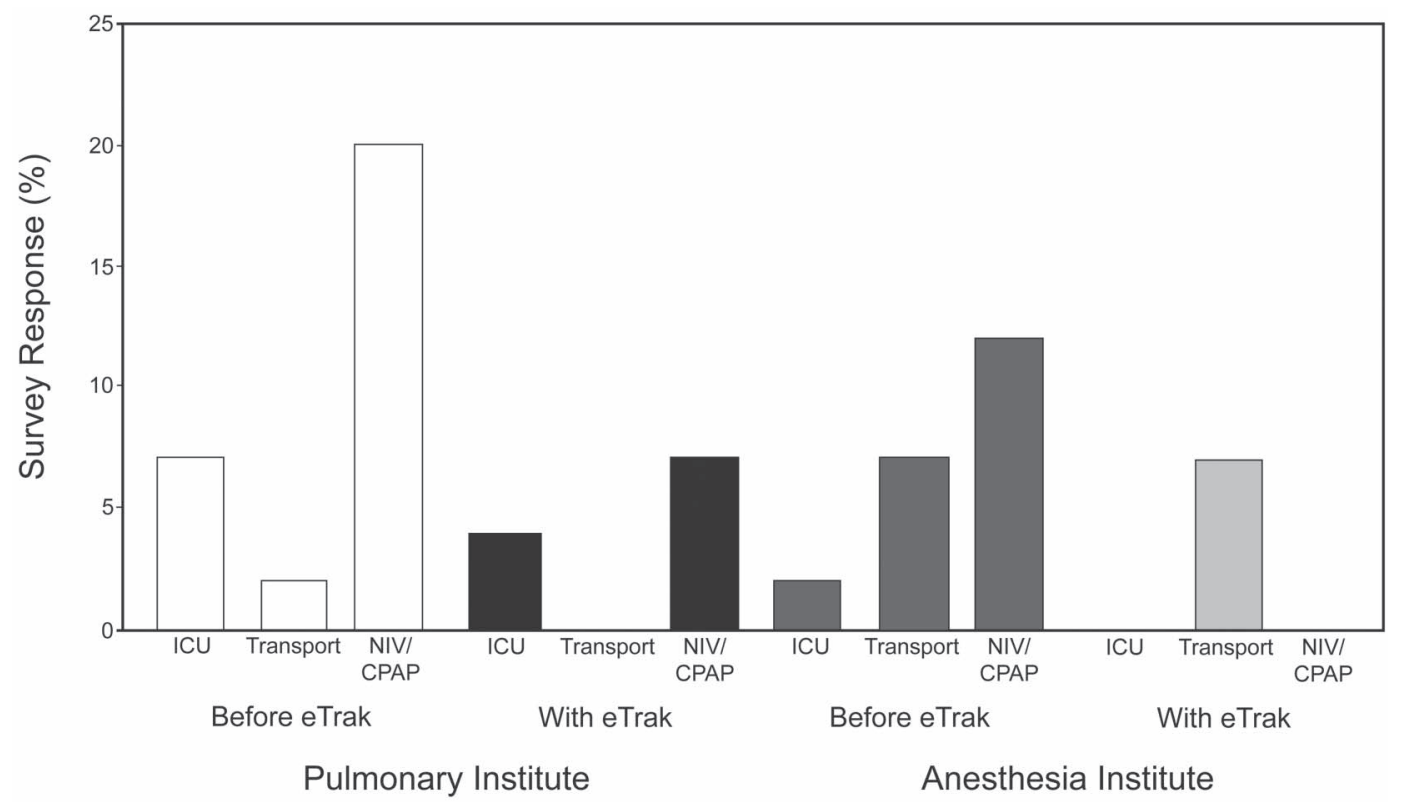

Fig. 5. Results of focused surveys of respiratory therapists in 2 departments, regarding the ease of finding equipment before versus after implementing the eTrak system. The values are the percentage of respondents who indicated that equipment was too hard to find.

surveyed before and again after implementing the eTrak system. The first survey was conducted during the first quarter of 2010 ( $n=69$ completed surveys) and the second survey was conducted during the second quarter of 2011 ( $n=55$ completed surveys). The results (Fig. 5) indicate a non-significant trend toward improved satisfaction in finding ICU ventilators, transport ventilators, and noninvasive bi-level ventilators/CPAP devices, with the largest change in the latter category.

Several challenges were experienced in implementing the eTrak system. For example, the initial set of RFID tags had a short battery duration, requiring adjustment of the software settings to minimize unnecessary activity of the tag (ie, too frequent signaling of device location). This initial struggle with poor battery duration decreased staff enthusiasm about the usefulness of the system. Some users reported problems with the software, citing difficulties with log-on procedures and occasional inaccuracy of the indicated device location. However, we are not sure to what extent this reflects user error versus software or hardware bugs.

Data in the eTrak system (eg, serial numbers and 12digit tag media access control address, a unique identifier assigned to network interfaces for intranet communication) must be manually entered into the system. In a large facility such as ours, this means hundreds of lines of data entry. The reliability of that data relies on mistake-free data entry. As the tag was placed on a machine, the person placing the tag transcribed the machine and tag information and sent that via e-mail to a designated person to be entered into eTrak. Although no such errors have been identified, the possibility for error is evident.

\section{Discussion}

Our early experience with an RFID healthcare equipment tracking system shows that our internally developed system significantly shortened the RT time needed to find a ventilator. Given the observed time savings $(15 \mathrm{~min} /$ ventilator), an RT seeking 4 ventilators over the course of an 8 -hour shift would save 1 hour (12.5\% time savings). Also, though it was a non-significant trend, we think the improved RT satisfaction may be important.

While our observations are preliminary, they extend the relatively sparse available experience with RFID in respiratory therapy. There are many reports of RFID use in healthcare in general. ${ }^{2-9}$ At Nemours Children's Hospital in Orlando, Florida, RFID supports the concept of a hospital having a single logistics center that monitors and runs operations. Interest in RFID technology seems high, as it can be applied beyond asset location to temperature and humidity monitoring and improving clinician workflow. Scattered reports also describe using RFID technology to detect retained surgical sponges, ${ }^{6,9}$ to monitor endotracheal tube position, ${ }^{8}$ and for recording operating room timestamps. ${ }^{7}$ However, except for a laboratory study that reported that RFID tags on ventilators can cause electromagnetic interference, ${ }^{4}$ we are unaware of any reports of using RFID technology to tag ventilators. Also, only a few reports describe hospital-wide application of RFID tech- 
nology. For example, Britton ${ }^{2}$ summarized the experience at 6 other institutions and a pilot experience at his hospital, the Royal Alexandra Hospital in Renfrewshire, United Kingdom. ${ }^{2}$ At the Bon Secours Health System in Virginia, RFID labeling of 12,000 pieces of equipment was undertaken at 3 separate hospitals, with reported benefits of decreasing by $30 \mathrm{~min}$ per shift the time that nurses spent searching for equipment, and decreasing equipment disappearance. At the Advocate Good Shepherd Hospital in Illinois and the Holy Name Society Hospital in New Jersey, RFID tracking programs were implemented (without statement of results), and at St Vincent's Hospital in Alabama, an RFID program was undertaken to track and manage surgical instruments (eg, for location, sterilization, and maintenance). Again, no specific data were cited regarding outcomes or the impact of the RFID program.

Roberti ${ }^{10}$ described the use of 1,900 active RFID tags in a 500-bed Georgia hospital to cover $75 \%$ of the million square feet of the facility. Whether or not ventilators were tagged was not stated. Taken together, the promise of RFID tagging of ventilators aligns with a larger trend of RFID tagging for inventory control and management in general, involving healthcare and other business sectors.

There have been several comprehensive reviews of the literature on RFID in healthcare. Wamba ${ }^{11}$ reviewed 22 articles in the Journal of Medical Systems from 1997 to 2011, and presented a list of future research directions. Mehrjerdi ${ }^{12}$ reviewed the cost of active and passive tags and identified technical and managerial problems for RFID applications, including reliability, identification range, and implementation costs. Yao et al ${ }^{13}$ reviewed RFID applications, perceived benefits, and barriers to adoption. They also described critical success factors and concluded that RFID technology in healthcare is still in its infancy, compared with RFID in other areas of application. Regarding implementation, Ting et al $^{14}$ reported that most of the literature focuses on the benefits of RFID in healthcare, with little attention to project management issues. Their paper provides a case study that illustrates the developmental framework and 23 critical issues in the preparation, implementation, and maintenance of an RFID project. They concluded that the task is complex and many challenges remain unaddressed, and they cited 5 areas for future research. Finally, Boulos and Berry ${ }^{15}$ reported a state-of-the-art review of real-time locating systems and technologies, including light, camera, infrared, sound, ultrasound, Bluetooth, Wi-Fi, and RFID. They listed 12 examples of mobile assets related to respiratory care that can be tracked.

Our data contribute to the available literature on RFID in healthcare in 4 ways. First, we report experience with a new, locally designed RFID system. Second, this RFID tracking program was implemented in a large academic medical center. Third, to our knowledge, ours is the first report of tagging ventilators and CPAP devices. Finally, we report quantitative data regarding the impact of the RFID program on the time needed to find equipment and employee satisfaction regarding equipment search time before versus after implementing the system.

Our recommendations about implementing an RFID system are:

- When designing the system and its use characteristics, set the active tag signaling to maximize battery duration. Short battery duration creates a time-intensive burden for system maintenance.

- Plan for system maintenance and monitoring needs.

- Roll out implementation in a phased way, with small groups going live sequentially.

- Plan for and design the metrics that will be used to assess system input so as to assure their availability before and after implementing the system.

\section{Limitations}

First, though the eTrak system is adaptable to a variety of settings, our study does not address the generalizability of our findings to other settings (eg, regarding the system's impact on locating equipment). Furthermore, our data regarding equipment-search time were based on a small sample from the main Cleveland Clinic hospital, but not from other hospitals in our system. A comparison with larger groups and between hospitals within our system will be the subject of further inquiry.

Second, as a pilot experience, our study necessarily leaves unanswered several important questions, such as: What would be the rate of adoption in settings other than that in which the system was developed? What is the costeffectiveness of RFID tracking? Though we did not conduct a robust analysis of cost-effectiveness, the savings of 1 hour of RT time per shift permits a crude estimate of the break-even point for adopting the eTrak system. At an average staffing of 100 RTs daily earning an average salary of $\$ 59,416$ per year, ${ }^{16}$ implementation of the eTrak system is associated with capturing $\$ 2,856.54$ of productive work per day, or $\$ 1,042,400$ annually.

Third, we present the number of total weekly log-ons to eTrak as an overall metric of utilization, recognizing that this number likely exceeds the number of unique individual users of eTrak (eg, a single user logging on twice would be responsible for 2 log-ons).

\section{Conclusions}

Overall, our early experience suggests that RFID tracking of ventilators and other RT equipment confers a desired benefit of more efficient equipment location and may 


\section{RADIO-FREQUENCY TRACKING OF RESPIRATORY EQUIPMENT}

enhance RT satisfaction. Further investigation is needed to clarify remaining unanswered questions, including the generalizability of these findings to other healthcare settings and the cost-effectiveness of RFID tracking.

\section{REFERENCES}

1. Wikipedia editors. Radio-frequency identification. http://en.wikipedia. org/wiki/Radio-frequency_identification. Accessed September 20, 2013.

2. Britton J. An investigation into the feasibility of locating portable medical devices using radio frequency identification devices and technology. J Med Eng Technol 2007;31(6):450-458

3. Ashar BS, Ferriter A. Radiofrequency identification technology in health care: benefits and potential risks. JAMA 2007;298(19): 2305-2307.

4. Van der Togt R, van Lieshout EJ, Hensbroek R, Beinat E, et al. Electromagnetic interference from radio frequency identification inducing potentially hazardous incidents in critical care medical equipment. JAMA 2008;299(24):2884-2890.

5. Wicks AM, Visich JK, Li S. Radio frequency identification applications in hospital environments. Hosp Top 2006;84(3):3-8.

6. Macario A, Morris D, Morris S. Initial clinical evaluation of a handheld device for detecting retained surgical gauze sponges using radiofrequency identification technology. Arch Surg 2006;141(7): 659-662.
7. Marjamaa RA, Torkki PM, Torkki MI, Kirvela OA. Time accuracy of a radio frequency identification system for recording operating room timestamps. Anesth Analg 2006;102(4):1183-1186.

8. Reicher J, Reicher D, Reicher M. Use of radio frequency (RFID) tags in bedside monitoring of endotracheal tube position. J Clin Monit Comput 2007;21(3):155-158.

9. Rogers A, Jones E, Oleynikov D. Radio frequency identification (RFID) applied to surgical sponges. Surg Endosc 2007;21(7):1235-1237.

10. Roberti M. Hospital saves by using RFID to bring asset management in-house. RFID J 2010. http://www.rfidjournal.com/articles/view? 7802. Accessed September 20, 2013.

11. Wamba SF. RFID-enabled healthcare applications, issues and benefits: an archival analysis (1997-2011). J Med Syst 2012;36(6): 3393-3398.

12. Mehrjerdi YZ. Radio frequency identification: the big role player in health care management. J Health Organ Manage 2011;25(5):490-505.

13. Yao W, Chu C, Li Z. The adoption and implementation of RFID technologies in healthcare: a literature review. J Med Syst 2012; 36(6):3507-3525

14. Ting SL, Kwok SK, Tsang AHC, Lee WB. Critical elements and lessons learnt from the implementation of an RFID-enabled healthcare management system in a medical organization. J Med Syst 2011;35(4):657-669.

15. Boulos MNK, Berry G. Real-time locating systems (RTLS) in healthcare: a condensed primer. Int J Health Geogr 2012;(11):25.

16. Registered respiratory therapists' salaries. US national averages. http://swz.salary.com/SalaryWizard/Registered-RespiratoryTherapist-Salary-Details.aspx. Accessed September 20, 2013. 